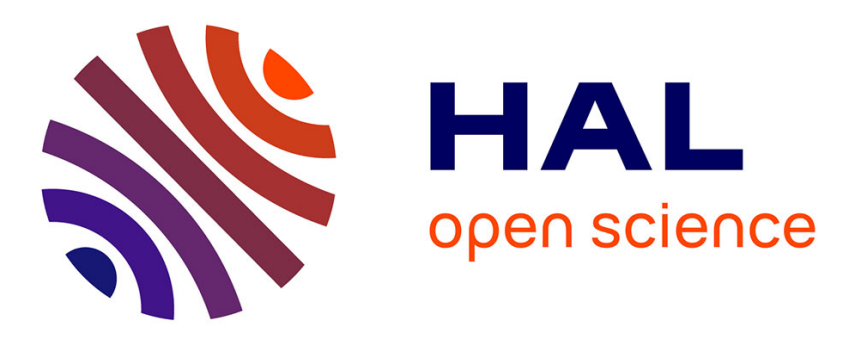

\title{
Effective dielectric constant of periodic composite materials
}

\author{
Bruno Sareni, Laurent Krähenbühl, Abderrahmane Beroual, Christian \\ Brosseau
}

\section{- To cite this version:}

Bruno Sareni, Laurent Krähenbühl, Abderrahmane Beroual, Christian Brosseau. Effective dielectric constant of periodic composite materials. Journal of Applied Physics, 1996, 80 (3), pp.1688-1696. 10.1063/1.362969 . hal-00140682

\section{HAL Id: hal-00140682 \\ https://hal.science/hal-00140682}

Submitted on 5 Jun 2019

HAL is a multi-disciplinary open access archive for the deposit and dissemination of scientific research documents, whether they are published or not. The documents may come from teaching and research institutions in France or abroad, or from public or private research centers.
L'archive ouverte pluridisciplinaire HAL, est destinée au dépôt et à la diffusion de documents scientifiques de niveau recherche, publiés ou non, émanant des établissements d'enseignement et de recherche français ou étrangers, des laboratoires publics ou privés. 


\section{Effective dielectric constant of periodic composite materials}

Cite as: Journal of Applied Physics 80, 1688 (1996); https://doi.org/10.1063/1.362969

Submitted: 28 November 1995 . Accepted: 28 April 1996 . Published Online: 17 August 1998

B. Sareni, L. Krähenbühl, A. Beroual, and C. Brosseau

\section{ARTICLES YOU MAY BE INTERESTED IN}

Effective dielectric constant of random composite materials

Journal of Applied Physics 81, 2375 (1997); https://doi.org/10.1063/1.364276

Modeling dielectric properties of composites by finite-element method

Journal of Applied Physics 92, 6743 (2002); https://doi.org/10.1063/1.1516837

Dielectric relaxation in dielectric mixtures: Application of the finite element method and its comparison with dielectric mixture formulas

Journal of Applied Physics 89, 8092 (2001); https://doi.org/10.1063/1.1372363

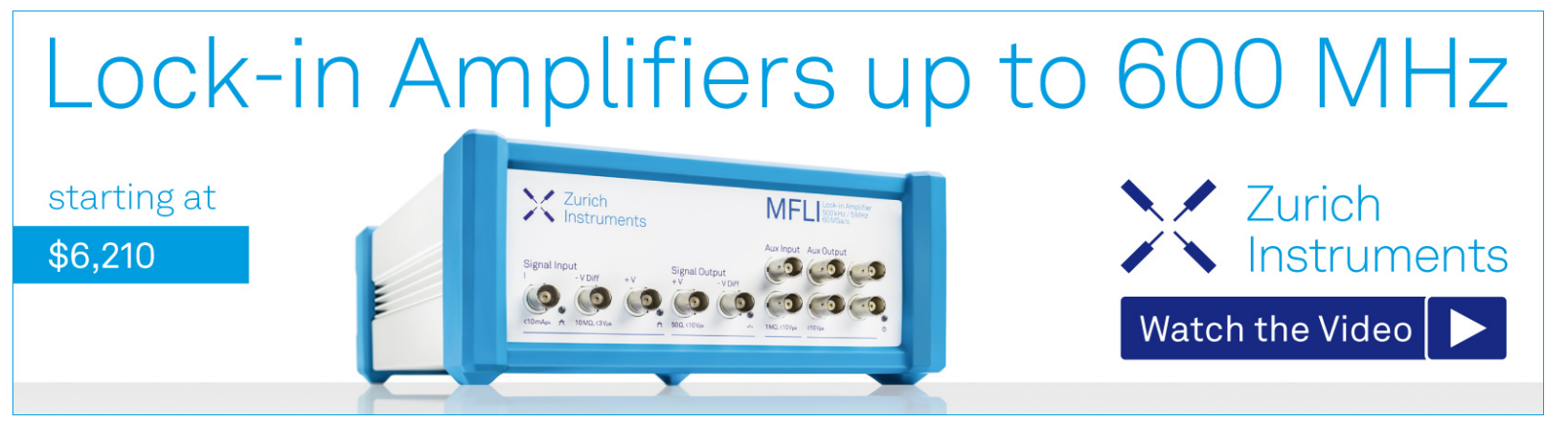




\title{
Effective dielectric constant of periodic composite materials
}

\author{
B. Sareni, L. Krähenbühl, and A. Beroual \\ Centre de Génie Electrique de Lyon, ${ }^{a)}$ Ecole Centrale de Lyon, B. P. 163, 36 avenue Guy de Collongue, \\ 69131 Ecully Cedex, France \\ C. Brosseau ${ }^{\text {b) }}$ \\ Département de Physique, Laboratoire d'Etude des Matériaux, Université de Bretagne Occidentale, \\ 6 avenue Le Gorgeu, B.P. 809, 29285 Brest Cedex, France
}

(Received 28 November 1995; accepted for publication 28 April 1996)

\begin{abstract}
We present computer simulation data for the effective permittivity (in the quasistatic limit) of a system composed of discrete inhomogeneities of permittivity $\epsilon_{1}$, embedded in a three-dimensional homogeneous matrix of permittivity $\epsilon_{2}$. The primary purpose of this paper is to study the related issue of the effect of the geometric shape of the components on the dielectric properties of the medium. The secondary purpose is to analyse how the spatial arrangement in these two-phase materials affects the effective permittivity. The structures considered are periodic lattices of inhomogeneities. The numerical method proceeds by an algorithm based upon the resolution of boundary integral equations. Finally, we compare the prediction of our numerical simulation with the effective medium approach and with results of previous analytical works and numerical experiments. (C) 1996 American Institute of Physics. [S0021-8979(96)06815-6]
\end{abstract}

\section{INTRODUCTION}

In recent years, extensive research has gone into studying the dielectric properties of heterogeneous materials. The reasons are not hard to find. On the one hand, it provides fundamental problems which are not completely answered, e.g. stochastic transport in disordered media, metal insulation transition. $^{1-3}$ On the other hand, industries such as aerospace, electronics and others, have continuously provided the impetus pushing the development of new materials in a wide variety of applications. These include fields as diverse as shielding enclosures, captive video disk units, electromagnetic absorbing materials, to cite but a few. ${ }^{4}$ The trend towards a wider variety of applications is almost certain to continue.

In these materials, an accurate prediction of the macroscopic dielectric behavior must account for the detailed internal structure of the composite, the dielectric and shape characteristics, the volume fractions and the spatial arrangement of the different components. The analytical solution requires us to compute the local fields inside the composite and their distortions caused by the inhomogeneities using a first principle approach, i.e. Maxwell's equations. In the general case of a spatially random structure, it appears as a formidable task to solve analytically this problem and these difficulties have led numerous groups to study the partial differential equations for the local fields using different computational techniques. $^{5-11}$ This originates from the fact that the effective permittivity of composite materials is basically an averaged property, where the average is taken over the ensemble of the realisations of disorder. Somewhat surprisingly, it should be emphasized in this context that numerical

\footnotetext{
a) Also at: UPRESA CNRS 5005.

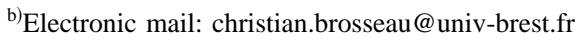

simulations which start from completely different descriptions of randomness may arrive at almost identical results. A program of investigation is currently underway, whose ultimate goal is to evaluate the effective permittivity of twophase composite materials in terms of the constituent properties and the internal structure of the mixture. The purpose of this paper is to develop a computer-simulation model based on the resolution of boundary integral equations with careful attention paid to the numerical evaluation of the local field. Although the method can be used to deal with arbitrary geometric forms of the inclusions and arbitrary spatial arrangements, calculations are confined, in this paper, to the special but important case of periodic composites. It should be regarded as a first step towards the ultimate goal outlined above. Actually, the cross-fertilization between computational and analytical work in this area is quickly growing. Extensive theoretical research has been focused in the study of the effect of microstructure on the effective permittivity in these materials. $^{13-17}$

The remainder of the paper is organized as follows. In Section II, we summarize the context of the problem. In Section III, we describe the principle of our numerical analysis. The boundary integral equation method, which is the cornerstone of this paper, will be reviewed in this section. In Section IV, we present results of different simulations with comparison with research reported by other authors. Our primary purpose is to determine the volume fraction dependence of the effective dielectric constant (in the quasistatic limit) of a composite material in which identical structures of constituent, say 1, are embedded in crystalline fashion in a matrix of constituent 2 . Our secondary purpose is to study the related issue of the influence of the geometric shape of the constituents. We pay particular attention to the issue of how the topological arrangement affects the effective permittivity. Finally, conclusions of the paper will be presented in Section V. 


\section{BACKGROUND}

The problem of determining the effective dielectric constant of heterogeneous two-phase materials has a long history. The origins of the modern concept of effective permittivity can be found in the scientific literature of the late nineteenth and early twentieth centuries. Particularly noteworthy early and pioneering contributions were made by Maxwell and Lord Rayleigh. For detailed historical reviews and discussions of the general subject of effective permittivity with numerous references inside, the reader may wish to consult Landauer ${ }^{3}$ and Tinga et al. ${ }^{14}$ In more recent times, important developments are found in the work of Shivola and Lindell. ${ }^{4}$ The medium under consideration will be characterized in the static limit, i.e. the spatial variation of the incident electric field is very large compared to the typical size of the heterogeneities in the medium. Note that in this paper, permittivity and dielectric constant are used synonymously.

Despite its effectiveness, the traditional boundary-value approach does not provide accurate values of $\epsilon$ at highvolume fraction of the inclusions because it neglects the correlations among the conductive inhomogeneities and does not contain information about the structure of the material, e.g. clustering effect. Moreover, this approach is restricted to nontouching inclusions.

There have already been a number of numerical studies which have found their way into electrostatics over the years. These include the random-walk method introduced by Schwartz and Banavar, ${ }^{7}$ and the multipole expansion of the field around inclusions to evaluate the local fields distortions, studied by Cukier et al. ${ }^{6}$ For completeness, we also mention that Felderhof et al. ${ }^{11}$ have proposed alternatives that use virial expansions and Torquato and Lado ${ }^{9}$ have also computed the effective permittivity of composite materials by applying bounding methods. Our calculations are very much in the spirit of the recent investigations reviewed by Ghosh and Azimi. ${ }^{23}$

\section{PRINCIPLE OF THE NUMERICAL APPROACH}

Let us turn to a brief presentation of the principle of our numerical analysis for describing the behavior of the electric field in composite materials. It is reasonable to start with the first principles of electrostatics, namely Laplace's equation, i.e. $\Delta V=0$ where $V$ is a potential distribution inside a spatial domain $\Omega$ with a density of charge equal to zero everywhere. The solution to this second order differential equation can be computed by applying the method of boundary integral equation (BIE). ${ }^{18,19}$ Upon using Green's theorem, we can write the local potential $V(M \in \Omega)$ in terms of $V(P)$ and of the normal derivative $\partial V / \partial n(P)$, with $P$ being any point on the boundary $\Sigma$ (with no overhangs) of $\Omega$ :

$$
V(M)=-\frac{4 \pi}{A} \int_{\Sigma}\left(V(P) \frac{\partial G}{\partial n}-G \frac{\partial V}{\partial n}(P)\right) d s,
$$

where $A$ stands for the solid angle under which the point $M$ sees the oriented surface $\Sigma, n$ is the normal unit vector oriented outward to $\Sigma, d s$ is a surface element of $\Sigma$ and $G$ denotes the Green function.

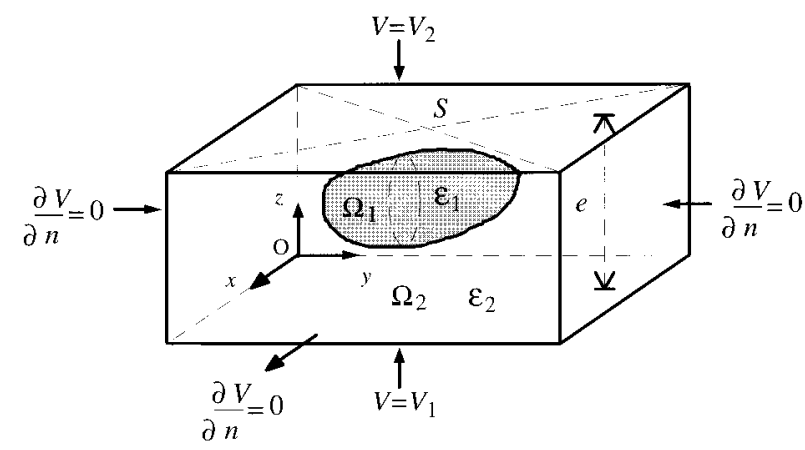

(a)

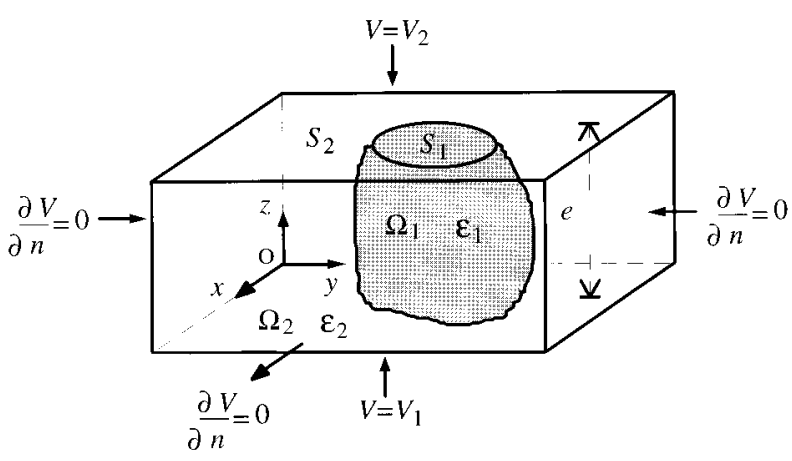

(b)

FIG. 1. Boundary conditions related to the configurations investigated in the numerical computation: (a) isolated particle of permittivity $\epsilon_{1}$, (b) fused particle of permittivity $\epsilon_{1}$.

To begin with we refer to the schematic representation of the configurations displayed in Fig. 1. We consider a twocomponent periodic composite that can be divided into elementary cells. The constituent of permittivity $\epsilon_{1}$ occupying the volume $\Omega_{1}$ is embedded in the region $\Omega_{2}$ of permittivity $\epsilon_{2}$. Absence of charge density will be tacitly assumed through our analysis. Given these assumptions, Eq. (1) leads to:

$$
V=-\frac{4 \pi}{A} \int_{\Sigma_{1}}\left(V \frac{\partial G}{\partial n}-\left.G \frac{\partial V}{\partial n}\right|_{1}\right) d s
$$

for domain 1 , and

$$
V=-\frac{4 \pi}{A} \int_{\Sigma_{2}}\left(V \frac{\partial G}{\partial n}-\left.G \frac{\partial V}{\partial n}\right|_{2}\right) d s
$$

for domain 2. Moreover, we have

$$
\left.\epsilon_{1} \frac{\partial V}{\partial n}\right|_{1}=\left.\epsilon_{2} \frac{\partial V}{\partial n}\right|_{2}
$$

by virtue of the conservation of the normal component of the electric displacement at the interface. Consequently, we have to solve the above two integral equations (2) and (3) to evaluate numerically the electrostatic potential distribution. For that purpose, the implementation of the BIE method consists in dividing the boundaries into finite elements and for each finite element, the calculation is carried out by interpolation of $V$ and $\partial V / \partial n$ with the corresponding nodal values: 


$$
\begin{aligned}
& V=\sum_{j} \lambda_{j} V_{j} \\
& \frac{\partial V}{\partial n}=\sum_{j} \lambda_{j}\left(\frac{\partial V}{\partial n}\right)_{j},
\end{aligned}
$$

where $\lambda_{j}$ denote the interpolating functions. The generation of these functions that are suited for our computational requirements and the detailed methodology that we employ in this work are similar to those reported at length elsewhere. $^{19,20}$ Following this way, integral equations are transformed in a matrix equation which is numerically solved using the boundary conditions on each side of the unit cell as displayed in Figures 1a and 1b. Then, the permittivity is obtained from the knowledge of the potential distribution and its normal derivative. We distinguish between two types of configurations for specifying the structure of the composite material.

In Fig. 1a, we have a single inclusion and thus, the medium of permittivity $\epsilon_{1}$ cannot intercept the sides of the parallelepipedic cell. In this case, the effective permittivity, in the direction corresponding to the applied field, is calculated using the following relation:

$$
\left.\int_{S} \epsilon_{2} \frac{\partial V}{\partial n}\right|_{2} d s=\epsilon_{z} \frac{V_{2}-V_{1}}{e} S,
$$

where $V_{2}-V_{1}$ denotes the slope of potential imposed in the $z$-direction, $e$ stands for the composite thickness in the same direction and $S$ denotes the surface of the unit cell perpendicular to the applied field.

In Fig. 1b the inclusion is allowed to intercept the sides of the parallelepipedic cell. In that case we must take into account the electric displacement flux through the area $S_{1}$ associated to the medium of permittivity $\epsilon_{1}$ to calculate the effective permittivity in the direction corresponding to the applied field. Then Eq. (6) is turned into

$$
\left.\int_{S_{2}} \epsilon_{2} \frac{\partial V}{\partial n}\right|_{2} d s+\left.\int_{S_{1}} \epsilon_{1} \frac{\partial V}{\partial n}\right|_{1} d s=\epsilon_{z} \frac{V_{2}-V_{1}}{e}\left(S_{1}+S_{2}\right),
$$

where $S_{1}$ and $S_{2}$ are the surfaces resulting from the intersection of the volumic regions of permittivity $\epsilon_{1}$ and $\epsilon_{2}$ respectively with the upper side of the unit cell, perpendicular to the applied field.

It should be noted that the BIE method gives an accurate description of the electric potential by taking into account edge and proximity effects even at low and high concentrations of inhomogeneities. Therefore, this numerical technique does not suffer from the disadvantages of the traditional boundary-value approach.

\section{RESULTS AND DISCUSSION}

We turn now to a discussion of numerical results concerning the static effective permittivity of periodic composite media as a function of the permittivities and the volume fraction of the constituent materials. Comparison with other numerical simulations and analytical equations for calculating the permittivity in heterogeneous media will be discussed.

The different geometries of the three-dimensional periodic composites, consisting of two lossless materials with dielectric constants $\epsilon_{1}$ and $\epsilon_{2}$, that we consider in this study are displayed in Fig. 2. The first subsection deals with effective permittivity of periodic arrays of dielectric spheres. The second concerns regular systems of dispersed ellipsoid. The third considers inclusions with cylinder shape (rods and discs). Finally, we examine how the effective permittivity can be affected by the type of the periodic arrangement.

\section{A. Composite with spherical inclusions}

Consider equal-sized spheres fixed in a simple cubic array, $a$ being the radius of the spheres. Figure 2 a shows a unit cell of the structure. For the purpose of simplicity, we assume, in the following, that all the lengths $(l, a)$ are dimensionless and that the side of the cell has the specific value $l=2$. It is worth noting that if $a \ll 1$ the particles act like isolated ones: they will experience only the external field and not the fields induced by the other particles. We call this case the isolated particle regime. Then, the permittivity of the medium can be described by the Maxwell-Garnett equation:

$$
\epsilon=\epsilon_{2} \frac{\epsilon_{1}+2 \epsilon_{2}+2 f\left(\epsilon_{1}-\epsilon_{2}\right)}{\epsilon_{1}+2 \epsilon_{2}-f\left(\epsilon_{1}-\epsilon_{2}\right)},
$$

where $f=\pi a^{3} / 6$ is the volume fraction of the scatterer phase in the mixture (see Fig. 2a). It is a relatively simple exercise to show that Eq. (8) can be also written in the ClausiusMossoti form:

$$
\frac{\epsilon-\epsilon_{2}}{\epsilon+2 \epsilon_{2}}=f \frac{\epsilon_{1}-\epsilon_{2}}{\epsilon_{1}+2 \epsilon_{2}} .
$$

What occurs when increasing the concentration $f$ ? The distance of separation between two spheres decreases and particles will experience the local fields induced by other particles. The volume fraction $f_{p}=\pi / 6 \cong 0.523$ corresponding to the limit of touching spheres $(a=1)$ is the maximum packing threshold. Beyond that concentration, the geometry can be described according to Shen et al. ${ }^{21}$ excluding six segments of sphere from the unit cell. In this fused particle regime, the volume fraction is analytically calculated for a value of the radius $a$ in the range $1 \leqslant a \leqslant \sqrt{2}$ :

$$
f=\frac{\pi a^{3}}{6}-\frac{\pi}{4}(a-1)^{2}(2 a-1) .
$$

By taking into account the symmetry of the unit cell in the two regimes described above, the geometry is further reduced to one-eighth of the microstructure for calculations by the BIE method. Our results are compared with those derived from Eq. (8) and with numerical data obtained by Mc Phedran ${ }^{5}$ et al. and Tao et al. ${ }^{8}$ for $\epsilon_{1}=3$ and $\epsilon_{2}=1$ (Figure 3). In that case, it appears that the values of $\epsilon$ computed by the BIE method agree satisfactorily with these previous calculations. One might argue that the agreement of the numerical data with Eq. (8), for $f>f_{p}$, is somewhat fortuitous. This is confirmed by Fig. 4 which shows the limits of the Maxwell-Garnett theory for volume fractions $f$ higher than 

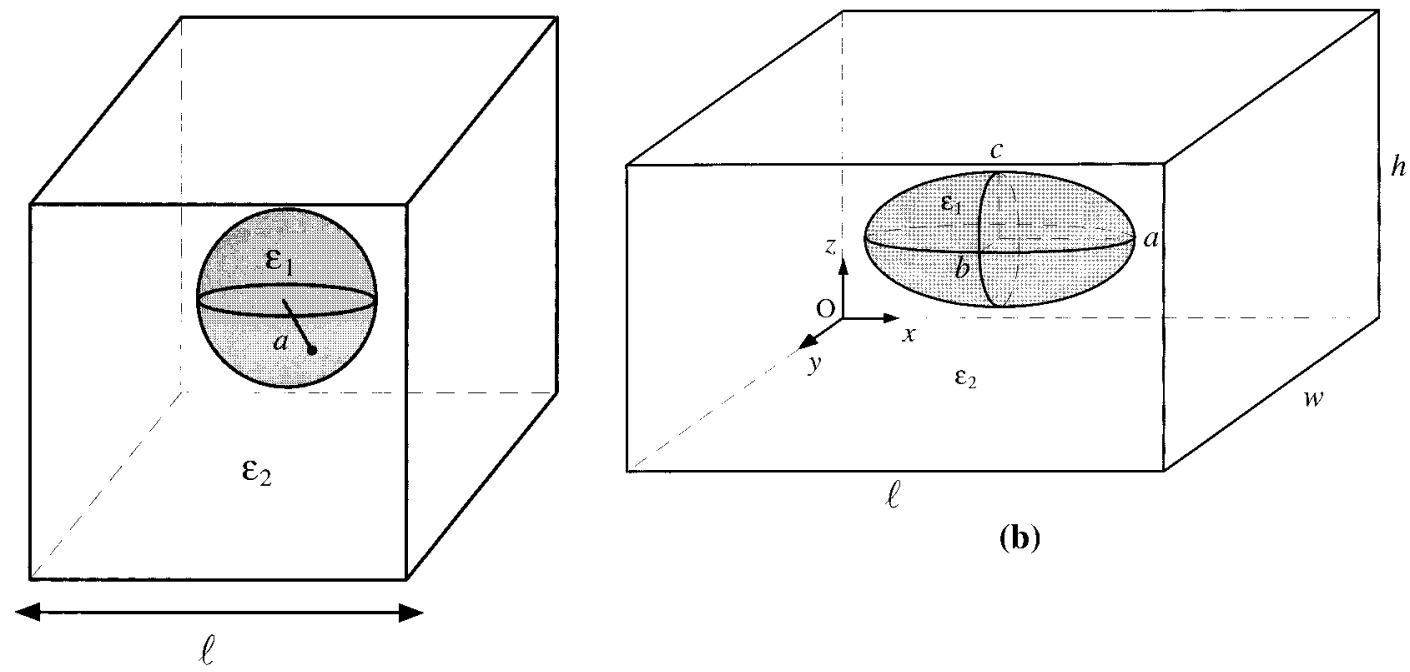

(b)

(a)
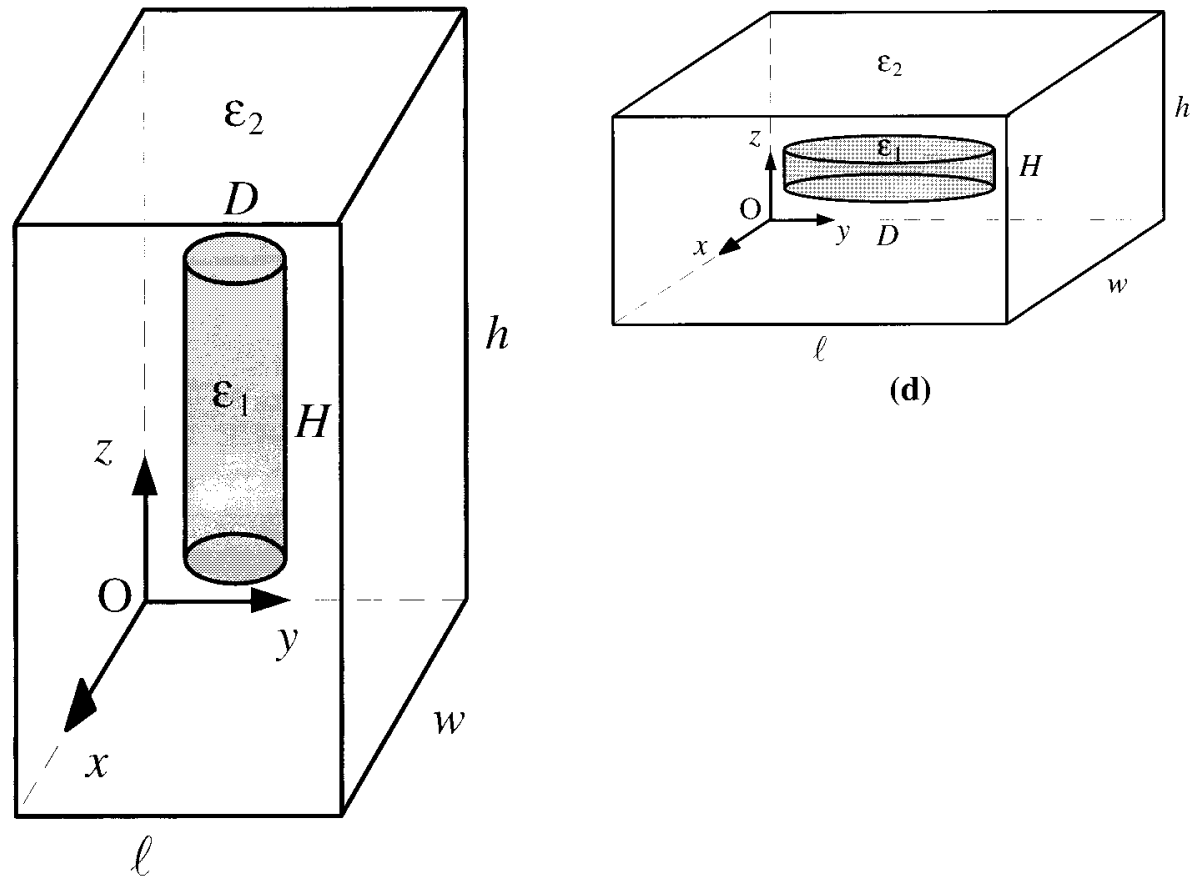

(d)

(c)

FIG. 2. The configurations investigated in the boundary element model computation. The volume fraction of material $\epsilon_{1}$ is $f$ : (a) sphere, (b) ellipsoid, (c) cylinder, (d) disc.

0.4 , when the ratio of the constituent permittivities is higher (we take $\epsilon_{1}=30$ and $\epsilon_{2}=1$ ). It is further interesting to note that our results are very close to those obtained by a Fourier expansion technique over the entire range of volume fraction $f .^{8,21}$

\section{B. Composite with ellipsoidal inclusions}

We examine now a system of ellipsoidal inclusions (Fig. 2b) which are regularly dispersed in a host medium. The unit cell can be described using the one obtained for spherical inclusions thanks to a tridimensional homothecy of ratio $(l, w, h)$, where $l, w$ and $h$ denote respectively the lengths of the sides of the parallelepipedic cell displayed in Fig. 2b. As can be seen in Fig. 2b, the medium is anisotropic and the effective permittivity component $\epsilon_{i}$ in the direction $i=x, y, z$ can be written as

$$
\epsilon_{i}=\epsilon_{2}+\frac{n \alpha_{i}}{1-L_{i} \frac{n \alpha_{i}}{\epsilon_{2}}},
$$

where $L_{i}$ and $\alpha_{i}$ are the depolarization factor and the polarizability in the direction characterized by the index $i$.

A standard result of electrostatics gives 


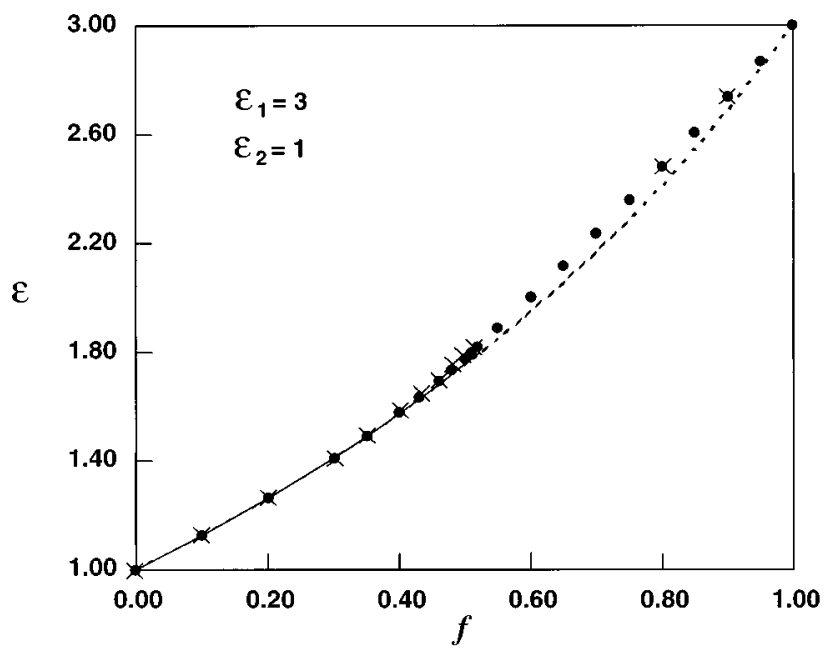

FIG. 3. Volume fraction dependence of the effective permittivity of the three-dimensional periodic composite displayed in Fig. 2a. Inclusions (permittivity $\epsilon_{1}=3$ ) are spherical and of volume fraction $f$ in a matrix of permittivity $\epsilon_{2}=1$. The full circles are obtained by the BIE method. The solid line is obtained from the results of Mc Phedran et al. (see Ref. 5) (x) are results of Tao et al. (see Ref. 8). The dashed curve corresponds to the Maxwell Garnet equation (Eq. (8)).

$$
L_{x}=\frac{a b c}{2} \int_{0}^{+\infty} \frac{d u}{\left(u+a^{2}\right) \sqrt{\left(u+a^{2}\right)\left(u+b^{2}\right)\left(u+c^{2}\right)}},
$$

where $a, b$ and $c$ denote the semiaxes of the ellipsoid. Note that $L_{y}$ and $L_{z}$ can be evaluated by interchanging $b$ and $a$, and $c$ and $a$ respectively. ${ }^{4}$

If the scatterers are sufficiently distant from each other, their polarizability can be deduced from the solution of the internal field of a dielectric ellipsoid in a quasistatic field

$$
\alpha_{i}=v 0\left(\epsilon_{1}-\epsilon_{2}\right) \frac{\epsilon_{2}}{\epsilon_{2}+\left(\epsilon_{1}-\epsilon_{2}\right) L_{i}}, \quad i=x, y, z,
$$

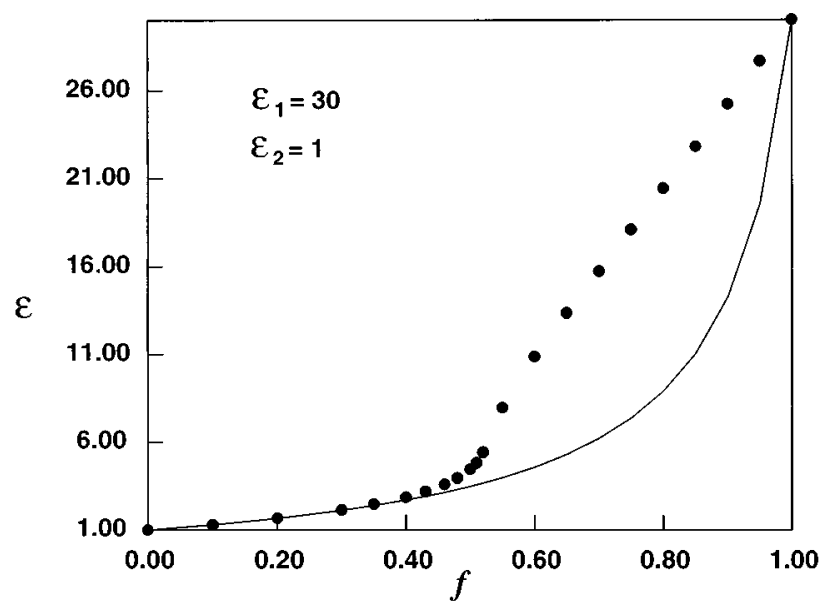

FIG. 4. Volume fraction dependence of the effective permittivity of the three-dimensional periodic composite displayed in Fig. 2a. Inclusions (permittivity $\left.\epsilon_{1}=30\right)$ are spherical and of volume fraction $f$ in a matrix of permittivity $\epsilon_{2}=1$. The full circles are obtained by the BIE method. The solid curve corresponds to the Maxwell-Garnet equation (Eq. (8)).

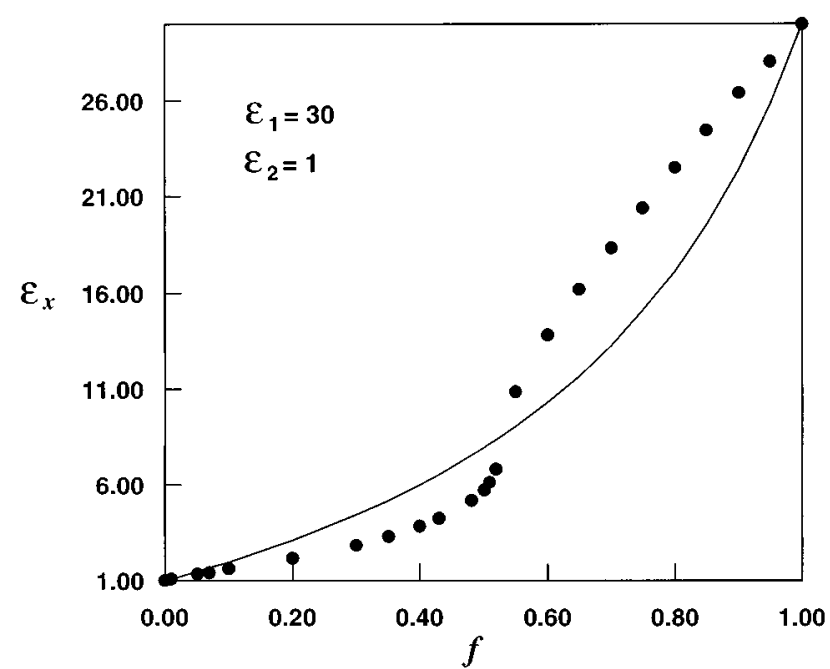

FIG. 5. Volume fraction dependence of the effective permittivity in the $x$ direction of the three-dimensional periodic composite displayed in Fig. $2 b$. Inclusions (permittivity $\left.\epsilon_{1}=30\right)$ are ellipsoid $(b=c=a / 4)$ and of volume fraction $f$ in a matrix of permittivity $\epsilon_{2}=1$. The full circles are obtained by the BIE method. The solid curve corresponds to Eq. (14) with a depolarization factor $L_{x}=0.0754$ (Eq. (12)).

where $v 0=\frac{4}{3} \pi a b c$ is the volume of the ellipsoid. ${ }^{4}$

By substituting Eq. (13) into (11), the effective permittivity of the medium can be expressed as

$$
\boldsymbol{\epsilon}_{i}=\epsilon_{2}\left(1+\frac{\left(\epsilon_{1}-\epsilon_{2}\right) f}{\epsilon_{2}+\left(\epsilon_{1}-\epsilon_{2}\right)(1-f) L_{i}}\right) i=x, y, z,
$$

where $f=n v 0=\frac{4}{3} \pi a b c / l w h$ is the volume fraction of the constituent 1 (See Fig. 2b). To simplify further the analysis, we first choose $b=c=a / 4$ (prolate spheroid) and we take the dimensions of the elementary cell as: $l=8$ and $w=h=2$. Figures 5 and 6 show a comparison of the numerical results

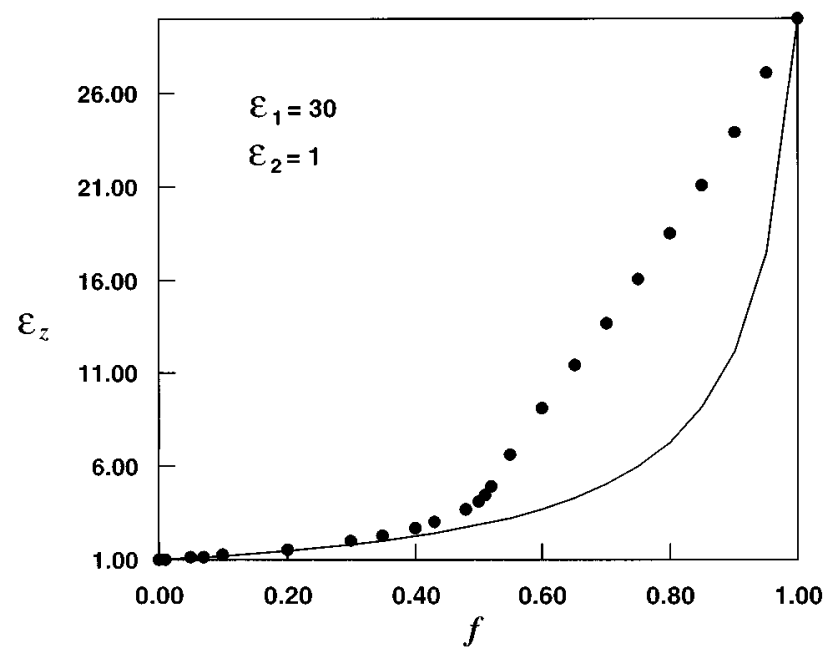

FIG. 6. Volume fraction dependence of the effective permittivity in the $z$ direction of the three-dimensional periodic composite displayed in Fig. 2c. Inclusions (permittivity $\left.\epsilon_{1}=30\right)$ are ellipsoid $(b=c=a / 4)$ and of volume fraction $f$ in a matrix of permittivity $\epsilon_{2}=1$. The full circles are obtained by the BIE method. The solid curve corresponds to Eq. (14) with a depolarization factor $L_{z}=0.4623$ (Eq. (12)). 


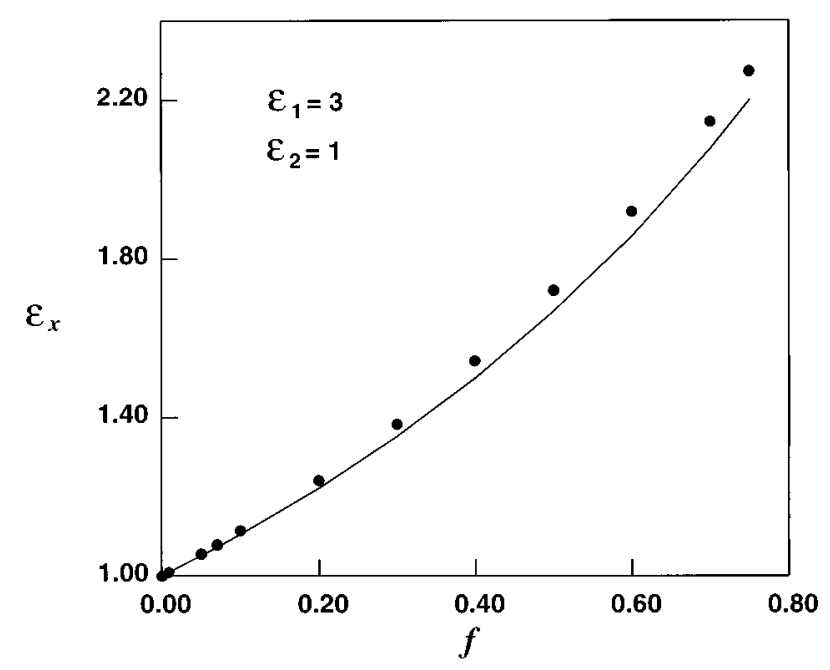

FIG. 7. Volume fraction dependence of the effective permittivity in the $x$ direction of the three-dimensional periodic composite displayed in Fig. 2c. Inclusions (permittivity $\epsilon_{1}=3$ ) are cylindrical and of volume fraction $f$ in a matrix of permittivity $\epsilon_{2}=1$. The full circles are obtained by the BIE method. The solid curve is obtained from Eq. (15).

obtained by the BIE method for the effective permittivity of the medium in the $x$ and $z$ directions respectively, $\left(\epsilon_{1}=30\right.$ and $\epsilon_{2}=1$ ) and those obtained from Eq. (14). They clearly show the limits of the analytical model for volume fractions $f$ higher than 0.2 particularly; the gap being important in the $x$ direction for which the interaction effect is stronger.

\section{Composite with cylindrical and discoidal inclusions}

We first consider the periodic composite displayed in Fig. 2c. By taking into account the symmetries of the unit cell, we need only to evaluate the permittivity in the $x$ and $z$ direction since $\epsilon_{y}=\epsilon_{x}$. The dielectric constant in the perpendicular direction to the cylinder axes is given by the Rayleigh's formula

$$
\epsilon_{x}=\epsilon_{2} \frac{\epsilon_{1}+\epsilon_{2}+f\left(\epsilon_{1}-\epsilon_{2}\right)}{\epsilon_{2}+\epsilon_{2}-f\left(\epsilon_{1}-\epsilon_{2}\right)} .
$$

Using Eq. (14) for prolate spheroid with $L_{x}=L_{y}=\left(\frac{1}{2}-\delta\right)$ and $L_{z}=2 \delta$ where $\delta \ll 1 \mathrm{Van} \mathrm{Beek}^{13}$ derived a general expression for the effective permittivity in the direction which is parallel to the cylinder axes

$$
\epsilon_{z}=\epsilon_{2}+\frac{1}{3} \frac{\left(\epsilon_{1}-\epsilon_{2}\right)\left(5 \epsilon_{a}+\epsilon_{1}\right)}{\epsilon_{a}+\epsilon_{2}},
$$

where $\epsilon_{a}$ denotes the apparent permittivity of the medium, i.e. "seen" outside by an inclusion. Its value differs from the permittivity of the host medium but must lie in the range $\epsilon_{2} \leqslant \epsilon_{a} \leqslant \epsilon_{z}$.

To make our simulation simpler, the geometry of the unit cell was characterized by a single parameter $a$ by taking: $H=8 a, D=a, l=w=1$, and $h=8$ (see Fig. 2c). The corresponding volume fraction of the constituent 1 is given by $f=\pi a^{3} / 4$. Figures 7 and 8 show data computed with the BIE method. At this point, a number of comments are in order. It is first interesting to observe that numerical data concerning

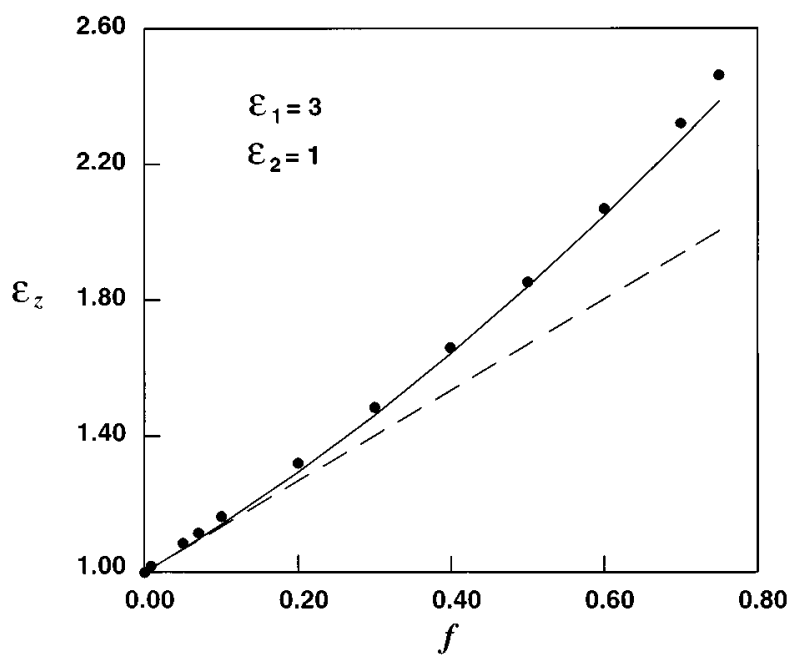

FIG. 8. Volume fraction dependence of the effective permittivity in the $z$ direction of the three-dimensional periodic composite displayed in Fig. 2c. Inclusions (permittivity $\epsilon_{1}=3$ ) are cylindrical and of volume fraction $f$ in a matrix of permittivity $\epsilon_{2}=1$. The full circles are obtained by the BIE method. The dashed and the solid lines are obtained from Eq. (16) with the apparent permittivities $\epsilon_{a}=\epsilon_{2}$ and $\epsilon_{a}=\epsilon$ respectively.

$\epsilon_{x}$ are well represented by Eq. (15) for inclusions of low permittivity $\left(\epsilon_{1}=3\right)$, while for high permittivity we have noted a significant departure from Eq. (15) at high concentration levels. As concerns $\epsilon_{z}$ we observe that numerical data for inclusions of low permittivity $\left(\epsilon_{1}=3\right)$ are well described by Eq. (16), taking an apparent permittivity identical to the effective permittivity in the parallel direction to the cylinder axis.

We turn next to the case of discoidal inclusions embedded in the host medium. The structure of the periodic composite has for unit cell the geometry of Fig. $2 \mathrm{~d}$.

In this case, it has been suggested that the components of the effective permittivity can be evaluated from the Wiener's formulae considering a periodic array of thin lamellae; $;^{3,13,14}$ $\epsilon_{z}$ is obtained by connecting the constituents in series.

$$
\frac{1}{\epsilon_{z}}=\frac{f}{\epsilon_{1}}+\frac{1-f}{\epsilon_{2}}
$$

while $\epsilon_{x}$ is deduced by connecting the constituents in parallel

$$
\boldsymbol{\epsilon}_{x}=f \epsilon_{1}+(1-f) \boldsymbol{\epsilon}_{2} .
$$

As in the first part of this subsection, the geometry of the unit cell is characterized by a single parameter: $H=a$, $D=10 a, l=w=10$, and $h=1$. The corresponding volume fraction of the constituent 1 is again given by $f=\pi a^{3} / 4$. The values of the effective permittivity obtained by the BIE method for inclusions of permittivity $\left(\epsilon_{1}=3\right)$ are displayed in Figs. 9 and 10. We can deduce from these figures that neither Eq. (17) nor Eq. (18) is able to correctly evaluate the permittivity of these structures.

\section{Lattices}

Up to now, we have only considered a simple cubic (sc) arrangement of the inclusions in the host matrix. Here, we discuss the influence of other types of cubic arrangements: 


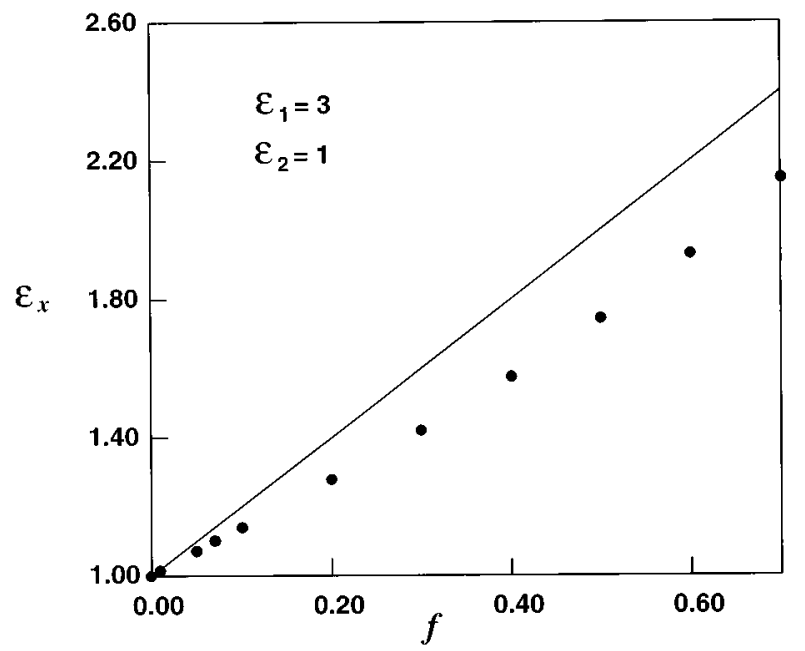

FIG. 9. Volume fraction dependence of the effective permittivity in the $x$ direction of the three-dimensional periodic composite displayed in Fig. 2 d. Inclusions (permittivity $\epsilon_{1}=3$ ) are discoidal and of volume fraction $f$ in a matrix of permittivity $\epsilon_{2}=1$. The full circles are obtained by the BIE method. The solid line is obtained from Eq. (19).

body centered (bcc) and face centered (fcc). We shall also consider crystalline lattices composed of perfectly conducting spheres $\left(\sigma_{1} \rightarrow+\infty\right)$ of radius $a$, the permittivity of the host medium being $\epsilon_{2}=1$. The volume fraction of the particles can be written as

$$
f=n_{0} \frac{4}{3} \frac{\pi a^{3}}{v_{0}},
$$

where $n_{0}$ is the number of particles per unit of internal structure and $v_{0}$ denotes the volume of the internal structure. The main characteristics of the different types of cubic lattices investigated are summarized in Table I. By computing the

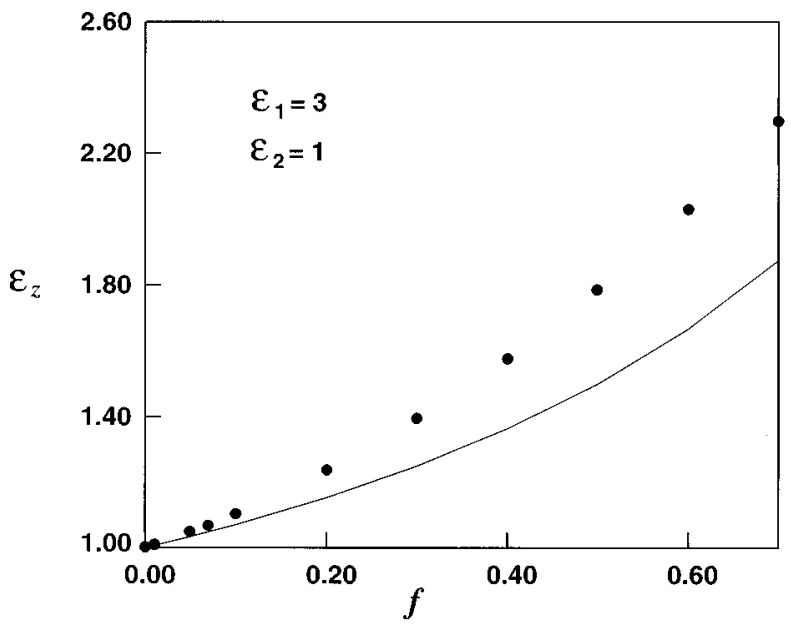

FIG. 10. Volume fraction dependence of the effective permittivity in the $z$ direction of the three-dimensional periodic composite displayed in Fig. 2d. Inclusions (permittivity $\epsilon_{1}=3$ ) are discoidal and of volume fraction $f$ in a matrix of permittivity $\epsilon_{2}=1$. The full circles are obtained by the BIE method. The solid curve is obtained from Eq. (18).
TABLE I. The main characteristics of the different types of cubic lattices. The internal structure, the number of particles per unit of internal structure $n_{0}$, the volume fraction $f$ of the conducting spheres of radius $a$, the radius $a_{p}$ and the concentration $f_{p}$ corresponding to the maximum packing threshold are function of the type of cubic lattices i.e. simple cubic (sc), bodycentered cubic (bcc) and face-centered cubic (fcc).

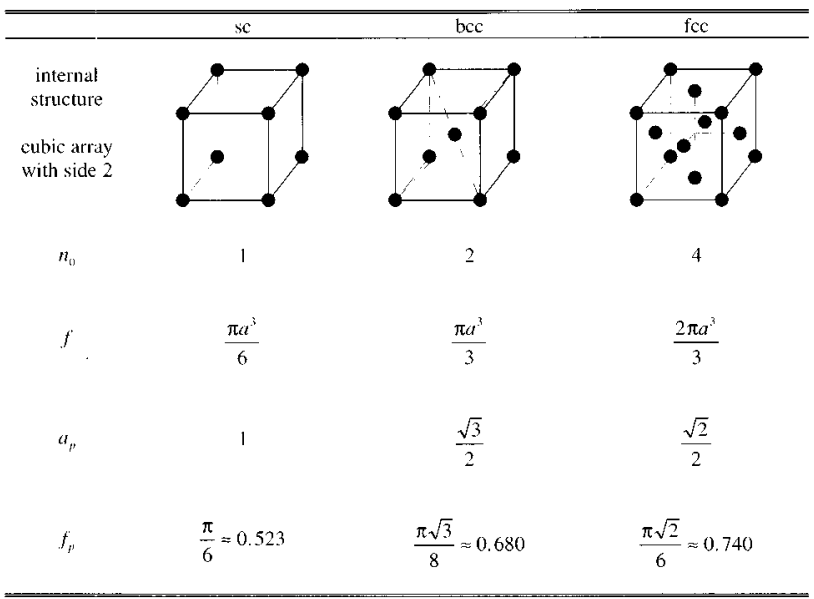

effective permittivity using the BIE method, we obtain identical results to those reported by Mc Phedran ${ }^{5}$ et al. and Doyle $^{12}$ with an accuracy of $10^{-3}$.

Next we turn to the case of hexagonal lattices composed of perfectly conducting spheres of radius $a$ embedded in a host medium of permittivity $\epsilon_{2}=1$. The geometry of the unit cell is displayed in Fig. 11. The number of particles per unit of internal structure is $n_{0}=1$, and the volume of the internal structure is $v_{0}=l^{2} h \sqrt{3} / 2$. This type of composite is anisotropic $\left(\epsilon_{x} \neq \epsilon_{z}\right)$ and we observe from the Fig. 12 that the permittivity strongly depends on the specific ratio $h / l$ for volume fractions $f$ higher than 0.1 .

\section{E. Comparison with previous approaches}

The above developments show that the BIE method can be used at high volume fractions, even for large permittivity contrast ratios between the background and the inclusions, i.e. when the mean-field analytical approaches are irrelevant to evaluate the effective permittivity of a composite material. We also made the comparison of our results with previously published analytical mixtures equations derived from heuristic assumptions which may be adapted for some composites
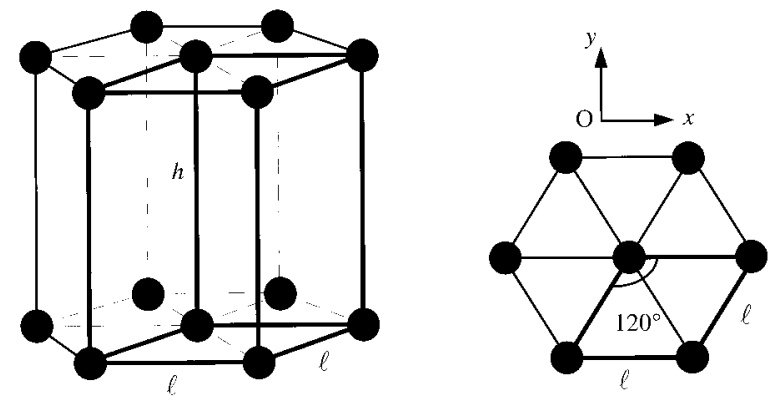

FIG. 11. Hexagonal lattice structure. 


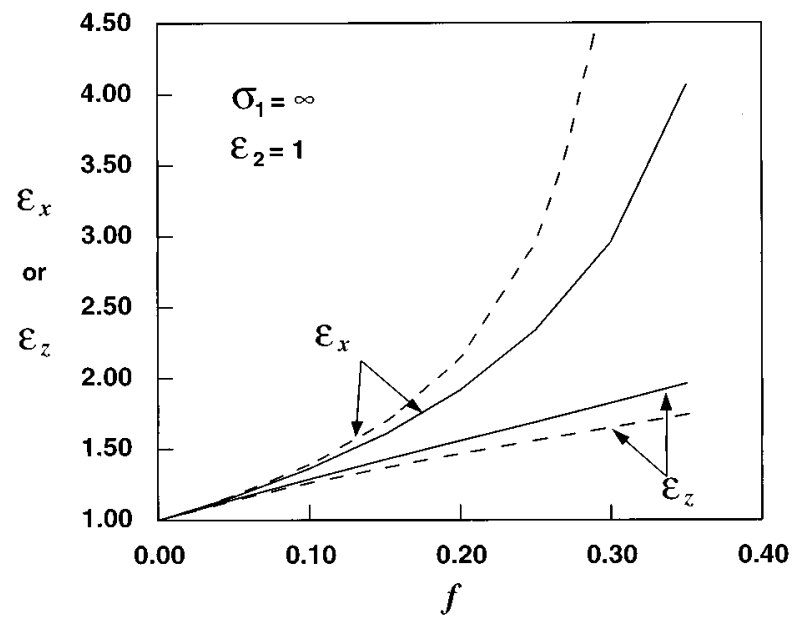

FIG. 12. Volume fraction dependence of the effective permittivity in the $x$ and $z$ directions of a hexagonal lattice composed of perfectly conducting spheres embedded in a host medium of permittivity $\epsilon_{2}=1$. The results obtained by the BIE method are presented for two values of the specific ratio $h / l$ : solid curve $(h / l=1.5)$ and dashed curve $(h / l=2)$.

but do not hold generally. We found that, for certain geometries, ranges of volume fraction and values of the dielectric constants of the two components, the differences between the BIE results and these equations may be small (e.g., Figs. 3 and 7), or not small (e.g., Figs. 4 and 6). Our method of calculation has three advantages. First, it is based on first principles so it can serve as a test for other approximate methods. Second, the BIE method can be easily realized in a computer algorithm. We made our calculations on a Hewlett-Packard 715/80 workstation. Third, it is not computationally time consuming. The CPU time for calculating the permittivity of a composite material with a certain concentration of inhomogeneities is about 20 minutes.

\section{CONCLUSIONS}

In summary, we have outlined an efficient and powerful computer-aided solution procedure based on the boundary integral equation method for the analysis of the static effective permittivity of two-constituent lossless media. Our numerical technique can be easily extended to multiphase structures of any shape.

Using such a framework, we have compared our results, in the range of permittivities investigated, with those given by mean-field approximations. Let us add that the numerical simulations have shown the limits of standard analytical models for high volume fractions of the dispersed phase, particularly when the ratio of the constituent permittivities is high. This is a most desirable development since in number of technological applications, the permittivity $\epsilon_{1}$ of inclusions is much larger than that of the matrix, e.g. carbon-black filled polymer composites. In this case interactions between particularly proximate neighbors cannot be neglected. These computations have also underlined the strong dependence of the geometric shape of the components and their spatial arrangement on the dielectric properties of the composite.
The calculated results have significant implications in the modeling of natural composites such as snow or reservoir rocks. While the results presented here seem to us encouraging, they represent only a first step in understanding how to describe the dielectric constant of these media. The code is currently being extended to treat composites with random distributions of inclusions. These capabilities will allow investigation of the technological problems that involve such randomness. Although we have confined ourselves, in this paper, to the static limit (the spatial correlation length of the material is smaller than the wavelength of the electric field), our ultimate goal is to investigate the range of absorption spectrum to provide an even finer discrimination among the geometries of heterogeneities. The study considered here can be extended to the case of complex dielectric constant $\left(\epsilon(\omega)=\epsilon^{\prime}(\omega)-i \epsilon^{\prime \prime}(\omega)\right.$, where $\omega$ is the angular frequency of the electromagnetic wave) to use the angular frequency for exploring the typical length scale $\xi$ of inhomogeneities. A complete description will be given in a forthcoming paper. Experiments dealing with the electromagnetic response of inhomogeneous materials have uncovered a varied phenomenology, whose interpretation presents challenging theoretical problems. It is also clear, that in order to deal with the issue of magnetodielectics, it is necessary to attack the full problem, i.e. $\mu_{1} \neq 1, \mu_{2} \neq 1$. In closing we also mention that since the differential equations for electrostatics and magnetostatics are identical, i.e. Laplace's equation for the potential, similar conclusions apply for the permeability of composite materials.

${ }^{1}$ D. J. Bergman and D. Stroud, in Solid State Physics, edited by H. Ehrenreich and D. Turnbull (Academic, New York, 1992), Vol. 46, pp. 178320. We also refer to the Proceedings of the Second International Conference on Electrical Transport and Optical Properties of Inhomogeneous Media [Physica A 157 (1989)].

${ }^{2}$ J. P. Clerc, G. Giraud, J. M. Laugier, and J. M. Luck, Adv. Phys. 39, 191 (1990).

${ }^{3}$ R. Landauer, in Electrical Transport Optical Properties of Inhomogeneous Media (Ohio State University Press, Columbus, OH, 1977); Proceedings of the First Conference on the Electrical and Optical Properties of Inhomogeneous Media, AIP Conf. No. 40, edited by J. C. Garland and D. B. Tanner (AIP, New York, 1978), p. 2; See also P. S. Neelakanta, Electron. Lett. 25, 800 (1989).

${ }^{4}$ A. H. Sihvola and I. V. Lindell, in Dielectric Properties of Heterogeneous Materials, edited by A. Priou (Elsevier, New York, 1992), p. 101.

${ }^{5}$ R. C. McPhedran and D. R. McKenzie, Proc. R. Soc. London Ser. A 359, 45 (1978).

${ }^{6}$ R. I. Cukier, S. Y. Sheu, and J. Tobochik, Phys. Rev. B 42, 5342 (1990); see also S. Y. Sheu, S. Kumar, and R. I. Cukier, ibid. 42, 1431 (1990).

${ }^{7}$ L. M. Schwartz and J. R. Banavar, Physica A (Amsterdam) 157, 230 (1989); see also Phys. Rev. B 39, 11965 (1989).

${ }^{8}$ R. Tao, Z. Chen, and P. Sheng, Phys. Rev. B 41, 2417 (1989).

${ }^{9}$ S. Torquato and F. Lado, Phys. Rev. B 33, 3370 (1986); 33, 5428 (1988).

${ }^{10}$ G. N. Milton, R. C. McPhedran, and D. R. McKenzie, Appl. Phys. 25, 23 (1981).

${ }^{11}$ B. U. Felderhof, G. W. Ford, and E. G. D. Cohen, J. Stat. Phys. 28, 135 (1982); 28, 649 (1982).

${ }^{12}$ W. Doyle, J. Appl. Phys. 49, 795 (1978).

${ }^{13}$ L. K. H. Van Beek, Progress in Dielectrics (Heywood, London, 1967), Vol. 7, p. 69.

${ }^{14}$ W. R. Tinga, W. A. G. Voss, and D. F. Blossey, J. Appl. Phys. 44, 3897 (1973).

${ }^{15}$ D. J. Bergman and Y. Imry, Phys. Rev. Lett. 39, 1222 (1977).

${ }^{16}$ D. A. G. Bruggeman, Ann. Phys. (Leipzig) 24, 636 (1935). 
${ }^{17}$ L. D. Landau and E. M. Lifshitz, Electrodynamics of Continuous Media (Pergamon, Oxford, 1960).

${ }^{18}$ P. K. Ghosh and M. E. Azimi, IEEE Trans. Dielect. 1, 975 (1994).

${ }^{19}$ L. Krähenbühl, Ph.D. thesis, Ecole Centrale de Lyon, France, 1983.
${ }^{20}$ C. A. Brebbia, The Boundary Element Method for Engineers (Pentech, London, 1980).

${ }^{21}$ L. C. Shen, C. Liu, J. Korringa, and K. J. Dunn, J. Appl. Phys. 67, 7071 (1990). 